archives

of thermodynamics

Vol. 38(2017), No. 4, 139-163

DOI: $10.1515 /$ aoter-2017-0029

\title{
Modeling of district heating networks for the purpose of operational optimization with thermal energy storage
}

\author{
MICHAŁ LEŚKO* \\ WOJCIECH BUJALSKI
}

Warsaw University of Technology Institute of Heat Engineering, Nowowiejska 21/25 00-665 Warszawa, Poland

\begin{abstract}
The aim of this document is to present the topic of modeling district heating systems in order to enable optimization of their operation, with special focus on thermal energy storage in the pipelines. Two mathematical models for simulation of transient behavior of district heating networks have been described, and their results have been compared in a case study. The operational optimization in a DH system, especially if this system is supplied from a combined heat and power plant, is a difficult and complicated task. Finding a global financial optimum requires considering long periods of time and including thermal energy storage possibilities into consideration. One of the most interesting options for thermal energy storage is utilization of thermal inertia of the network itself. This approach requires no additional investment, while providing significant possibilities for heat load shifting. It is not feasible to use full topological models of the networks, comprising thousands of substations and network sections, for the purpose of operational optimization with thermal energy storage, because such models require long calculation times. In order to optimize planned thermal energy storage actions, it is necessary to model the transient behavior of the network in a very simple way - allowing for fast and reliable calculations. Two approaches to building such models have been presented. Both have been tested by comparing the results of simulation of the behavior of the same network. The characteristic features, advantages and disadvantages of both kinds of models have been identified. The results can prove useful for district heating system operators in the near future.
\end{abstract}

${ }^{*}$ Corresponding Author. Email: mlesko@itc.pw.edu.pl 
Keywords: Thermal energy storage; District heating; Smart district heating; Optimization

\section{Introduction}

The aim of this document is to present the topic of modeling district heating systems in order to enable optimization of their operation, with special focus on thermal energy storage. The article is dedicated to energy storage in the pipelines, by shaping the supply temperature of water, rather than to utilization of hot water tanks.

Recent changes on energy markets, especially growing variations of electricity prices during the day, and the need to use combined heat and power (CHP) plants for balancing the electrical grid, make the value of heat delivered to DH systems very unstable. At the hours when the price of electricity is very high, or the CHP plant is called to production by the transmission system operator, the cogenerated heat can be considered a by-product available at zero cost. At some other hours, due to low electricity prices or the need to switch on a peak boiler, the marginal cost of heat at the CHP plant becomes much higher than the price at which it is sold to customers. This situation makes it very attractive to consider all possible ways of thermal energy storage, in order to maximize the total financial benefits from production of a CHP plant, at the same time delivering the heat to customers when they need it.

There are three basic options for thermal energy storage in DH: using hot water tanks, using thermal inertia of buildings and using the thermal inertia of pipelines. Among these three, using the thermal inertia of pipelines is the one which requires no additional investment and no remote control of substations - which makes it a very attractive solution for virtually all $\mathrm{DH}$ networks. In most DH systems this solution is already utilized, but the actions of overheating the network are typically done based on the experience of operators, without thorough calculation of costs and benefits. One of the reasons for this is the fact, that calculation of actual benefits at the CHP plant and of the effect on the heat losses in the network requires advanced models of the system. This article is dedicated to a part of this challenge - modeling of the network. The goal is not to make a large and precise thermo-hydraulic model, based on the real topology of actual grid. Such models, using Geographical Information System (GIS) data, are commercially available. However, their precision comes at the cost of calculation 
times, which in general make them not useful for optimization of heat production with the use of thermal energy storage. Such purpose requires very simple and fast models, compatible with reliable solvers, which in turn will make it possible to choose a proper scenario among thousands of possibilities, and present it to the operators before it is too late for decisions. The two simple approaches presented in current work are aimed at answering that need.

\section{Current state of research}

There are many works dedicated to the topic of optimization of CHP production and modeling of DH systems. A solution to the general problem of unit commitment in power production planning under the assumption of linear models of production units has been presented by Viana and Pedroso in [1]. Badyda [2] has described the general model of a backpressure extraction turbine. Interesting methods formodeling CHP units have been presented in [3] and [4]. Fonseca and Schneider [5] have described the results of modeling a particular CHP plant with different approaches. Ziębik et al. [6] have performed modeling of a CHP plant based on a technological diagram, despite very limited access to operational data. Bujalski [7] has proposed a solution for optimal scheduling of a CHP plant operation, with the use of a storage tank. Fazlollahi et al. [8] have proposed a procedure for optimization in DH on the stage of design and operation. Ziębik and Gładysz [9] have proposed an empirical formula for determination of the optimal value of the share of cogeneration in cogeneration systems with thermal storages. There are many works about optimization and controlling seasonal storages (STES) $[10,11]$. The topic of utilization of demand side management in space heating for the purpose of optimization has been also discussed in several works $[12,13]$. Hydraulic modeling of DH networks (in steady state) has been described in [14]. Thermo-hydraulic modeling of district heating and cooling networks was the topic of numerous articles such as [15]. Basciotti et al. [16] have presented a model for finding an optimal profile of supply temperature in a small district heating network. Similar work has been done by Benonysson et al. [17]. All these works describe particular solutions and their successful implementation. None of them presents a global optimization of a large DH system, including the possibility of thermal energy storage in a hot water tank, with the use of pipelines and with the use of thermal inertia of buildings at once. The 
objective of current study is to make a step towards such global optimization, by clear presentation of one part of such solution, i.e., models of the network. Rather than presenting a particular model, this article aims at a clear description and comparison of two possible approaches, in order to identify their advantages and disadvantages, which can help in choosing a proper solution for future projects dedicated to global optimization in $\mathrm{DH}$.

\section{Modeling a district heating network}

A DH system comprises heat sources, pipelines, pumping stations, storage tanks, valves and buildings with their substations. There are already comprehensive models and commercial solutions for simulation and optimization of operation of CHP plants. The topic of modeling the inertia of buildings has been described in [13]. In this article, the focus is on district heating networks.

District heating network pipelines are insulated steel tubes of a diameter between $20 \mathrm{~mm}$ and $1200 \mathrm{~mm}$ (or even more). It is natural to subdivide network into sections, with each section representing a continuous path of tubes characterized by the same diameter, roughness, insulation parameters and water mass flow. Typically it means that sections are divided by nodes representing substations, valves, heat production plants, pumping stations, storage tanks, etc.

\subsection{Hydraulic calculations}

Due to the size of the networks, in case of district heating it is not feasible to use precise methods from the field of fluid dynamics (e.g., finite element method) for hydraulic simulations. Instead, the calculations are based on simple equations describing linear pressure losses and local losses, which can be found, e.g., in [18]. Based on such equations and on the mass balance in every node, it is possible to determine pressure and water flow in every point of a network, given the boundary conditions (absolute pressure at one point and pressure differences at every pump or controlling valve). In case of small networks with tree structure such calculations are very simple, in case of large grids including ring connections usually iterative approach is required. 
Proper hydraulic state of the network means that:

- pressure difference between supply and return line is above the defined minimum at all the substations (it means that no regulation valve must be fully open in order to reach the desired heating power) and below the maximum which would be dangerous for the valves,

- at no point in the network the pressure drops below the global minimum limit (at which there would be a risk of underpressure resulting in air entering the pipelines through every untight connection),

- at no point in the network the pressure exceeds the global maximum limit (at which there would be a risk of damage to the pipelines).

At every moment of DH network operation, flow demands at substations and the grid configuration determine the optimal pumping scheme (power and pressure increase at particular available pumping stations) which would lead to proper hydraulic state. Finding that scheme can be done in realtime (because there are no delays) and in practice it is separated from the heat production planning. The plan of production (including supply temperature profiles) is done assuming that the pumps will allow reaching desired flows at all substations, and then the pumping control is performed in order to meet that assumption.

As the topic of current paper is strictly related to thermal energy storage, the main focus here will be on thermal models, which in fact define the delays and thermal inertia in a DH network, utilized for load shifting.

\subsection{Thermal calculations}

Thermal model of the network is needed for simulation of temperature propagation in the system. For given profile of the supply temperature at the heat source, outdoor temperature, heating power demands and return temperatures (or temperature drops) at all substations, assuming proper hydraulic state of the network, the thermal model should allow to simulate all the temperatures in the network and flows of $\mathrm{DH}$ water. As a result, total heating power at the heat source is determined (by calculation of total flow and return temperature at the heat plant), and heat losses can be calculated (based on temperature drops and flows in the pipelines).

Heat transmission is the most important phenomenon to account for. In reality, the heat is lost from both supply and return line to the ground, and also transmitted from the supply to return line. The scale of heat transfer 
depends on many factors such as state of the insulation (which varies over time), type of the ground, water speed, etc. As the heat transfer coefficients are temperature dependent, a perfect model would be very complicated and non-linear, making it extremely difficult to use for optimization purposes. It is crucial for system-scale optimization to build simple, preferably linear, models, which will still be able to reflect the actual behavior of the network.

In the most general form, the heat loss from water in a pipeline is proportional to the temperature difference between inside and outside, divided by the sum of thermal resistances of pipeline and insulation layers [18]

$$
\dot{Q}_{\text {loss }}=\frac{\left(T_{\text {water }}-T_{\text {out }}\right)}{\sum R}=-m_{\text {water }} c_{p} \dot{T}_{\text {water }},
$$

where: $\dot{Q}_{\text {loss }}$ - time derivative of heat loss, $T_{\text {water }}$ - temperature of the water in the pipeline, $T_{\text {out }}$ - ambient temperature, $\sum R$ - sum of thermal resistances, $m_{\text {water }}$ - mass of water inside the pipeline, $c_{p}$ - specific heat of water, $\dot{T}_{\text {water }}$ - time derivative of temperature of the water in the pipeline.

Based on that, assuming constant resistances, constant amount of water (in the pipeline or in the volume of interest) and constant specific heat, the simplest (and most convenient in case of very large models) equation for the pace of temperature drop can be written:

$$
\dot{T}_{\text {water }}=-x_{\text {loss }}\left(T_{\text {water }}-T_{\text {out }}\right),
$$

where: $x_{\text {loss }}$ - the heat loss coefficient for a given pipeline or volume of interest.

Two very general approaches to thermal calculations of the network are possible:

1. Focused on particular pipeline sections, treating them as objects which experience heat losses to the surroundings, water inflow and water outflow.

2. Focused on particular volumes of water, treating them as objects which travel through the pipelines and cool down through their walls. 


\subsection{Modeling the network with the focus on pipeline sections}

The energy balance of a pipeline section can be written as

$$
\triangle E=m_{\text {in }} c_{p} T_{\text {incoming }}-m_{\text {out }} c_{p} T_{\text {outgoing }}-Q_{\text {loss }},
$$

where: $\triangle E$ - change of total energy in the pipeline section, $m_{i n}$ - mass of water which has entered the pipeline, $T_{\text {incoming }}$ - temperature of water which has entered the pipeline, $m_{\text {out }}$ - mass of water which has left the pipeline, $T_{\text {outgoing }}$ - temperature of water which has left the pipeline, $Q_{\text {loss }}$ - amount of heat lost to the surroundings.

As the mass of water in the pipeline does not change, Eq. (3) after differentiation can be rewritten as:

$$
m_{\text {water }} c_{p} \dot{T}_{\text {in section }}=\dot{m}_{\text {flow }} c_{p}\left(T_{\text {incoming }}-T_{\text {outgoing }}\right)-\dot{Q}_{\text {loss }},
$$

where: $\dot{T}_{\text {in section }}$ - time derivative of the temperature in the pipeline section, $\dot{m}_{\text {flow }}$ - flowrate of incoming water, equal to the flowrate of the outgoing water.

In reality, a pipeline section can contain water at variable temperature, not possible to describe with a simple continuous function. It is a result of supply temperature changes at the heat source, water mixing (especially in return lines) and cooling down of the water. It is not feasible to build a model which will divide every pipeline section into elements characterized by uniform $\mathrm{DH}$ water temperature, and such division would have to vary in time. The most reasonable approach for simplified modeling of the whole network as an aggregation of pipeline sections is to assume uniform temperature in each section at every time step, and then calculate heat losses from each section at every time step which determines the temperature drops. The assumption is equivalent to full mixing of water within each pipeline section, which is far from reality for longer pipeline sections. However, it allows to simulate the thermal situation in the network in a simple way and without violation of energy balance principles.

Assuming uniform temperature in a pipeline section, considering Eqs. (1) and (2), Eq. (4) yields

$$
\dot{T}_{\text {in section }}=\frac{\dot{m}_{\text {flow }}}{m_{\text {water }}}\left(T_{\text {incoming }}-T_{\text {outgoing }}\right)-x_{\text {loss }}\left(T_{\text {water }}-T_{\text {out }}\right) .
$$


Based on that formula, a simple equation for fast calculation of water temperature in a pipeline section, in discrete time steps, can be written

$$
T_{t+\triangle t}=T_{t}+\frac{\dot{m}_{\text {flow }} \triangle t}{m_{\text {water }}}\left(T_{\text {incoming }}-T_{t}\right)-x_{\text {loss }}\left(T_{t}-T_{\text {out }}\right) \triangle t,
$$

where: $T_{t}$ - temperature of the water in the section at time $t, \Delta t$-time step.

Equation (6) is a base for thermal models assuming full mixing of water in every pipeline section. Their accuracy is limited, however by increasing the number of pipeline sections and adjusting the time step, they can be calibrated to bring satisfying results.

\subsection{Modeling whole network as one energy buffer}

A specific and probably the simplest model of the network is in fact a model with the focus on pipeline sections, but assuming that whole network is just one pipeline section (or more precisely, one energy buffer). It allows to track the state of network (in terms of charging/discharging with overheated water) and its influence on heat losses without considering the topology of the network. It can be used for preliminary calculations on any network, without preparations - it is enough to know some global coefficients (which are normally easy to compute based on historical data) and total flow. Equation (6) can be rewritten for such a model in the following form (describing the average supply temperature in whole network and the average return temperature in whole network, equal to the return temperature coming to the heat source):

$T_{\text {sup }, t+\triangle t}=$

$$
T_{\text {sup }, t}+\frac{\dot{m}_{\text {flow }} \triangle t}{m_{\text {water }}}\left(T_{\text {sup } C H P, t \rightarrow t+\triangle t}-T_{\text {sup }, t}\right)-x_{\text {loss }}\left(T_{\text {sup }, t}-T_{\text {out }}\right) \triangle t,
$$

$T_{\text {ret }, t+\triangle t}=$

$$
T_{\text {ret }, t}+\frac{\dot{m}_{\text {flow }} \triangle t}{m_{\text {water }}}\left(T_{\text {ret } S S T, t \rightarrow t+\triangle t}-T_{\text {ret }, t}\right)-x_{\text {loss }}\left(T_{\text {ret }, t}-T_{\text {out }}\right) \triangle t,
$$

where: $T_{\text {sup }, t}, T_{\text {ret, } t}$ - temperature of supply and return, at the time $t$, $T_{\text {sup } C H P, t \rightarrow t+\triangle t}, T_{\text {ret } S S T, t \rightarrow t+\triangle t}$ - average temperature of supply at the heat source and average temperature of return at the substations, over the time between $\mathrm{t}$ and $t+\Delta t$. 
It can be seen especially in the formula for return temperature (which at given flow and supply temperature determines total heating power of the heat plant), that this approach can give wrong results for long networks, where the average return temperature can be far from the return temperature reaching the heat source.

\subsection{Modeling the network with the focus on volumes of water}

In reality the water mixing in the supply pipelines of a DH network is limited. In order to reflect this fact, an approach with the focus on volumes of water can be proposed. In this approach, pipeline sections are considered only as a frame in which volumes of water ('waves') travel. But the heat losses are calculated for each 'wave' separately. By tracing the 'waves' in the network, it is possible to determine the temperature of $\mathrm{DH}$ water at every point of it and at every moment. At every time step, the temperature of water in the 'wave' is decreasing, based in Eq. (2):

$$
T_{\text {water }, t+\triangle t}=T_{\text {water }, t}-x_{\text {loss }}\left(T_{\text {water }, t}-T_{\text {out }, t}\right) \triangle t .
$$

If the water 'wave' reaches a split of pipelines (e.g., at the supply side of a substation), it must be divided in the model into two 'waves' (e.g., one flowing further in the network and one flowing through the substation to the net of return pipelines). If two waves reach a joint of pipelines (e.g., at the return side of a substation), they must be combined into one, new 'wave' at their weighted average temperature (e.g., a mixture of water returning from the substation and water flowing in the return lines at the location of the substation). The principle is illustrated in Fig. 1.

The 'wave' model seems to be the closest to reality, however it neglects the fact that there is some mass and heat exchange in the pipelines. The main drawback of that model is the fact that it is relatively complicated. Unlike in the approach focused on network sections, in the 'wave' model the number of elements on which the equations need to be applied, and their volume, are variable (as the 'waves' split and mix). This makes it more difficult to implement, and more time consuming to calculate. It is also crucial in this model, that the topology reflects the real behavior of the network, since the mechanism is based on tracing the flow of water volumes produced at different moments. On the other hand, replicating full topology of a large network would make a 'wave' model too slow for optimization calculations. A reasonable simplification is crucial, in order 


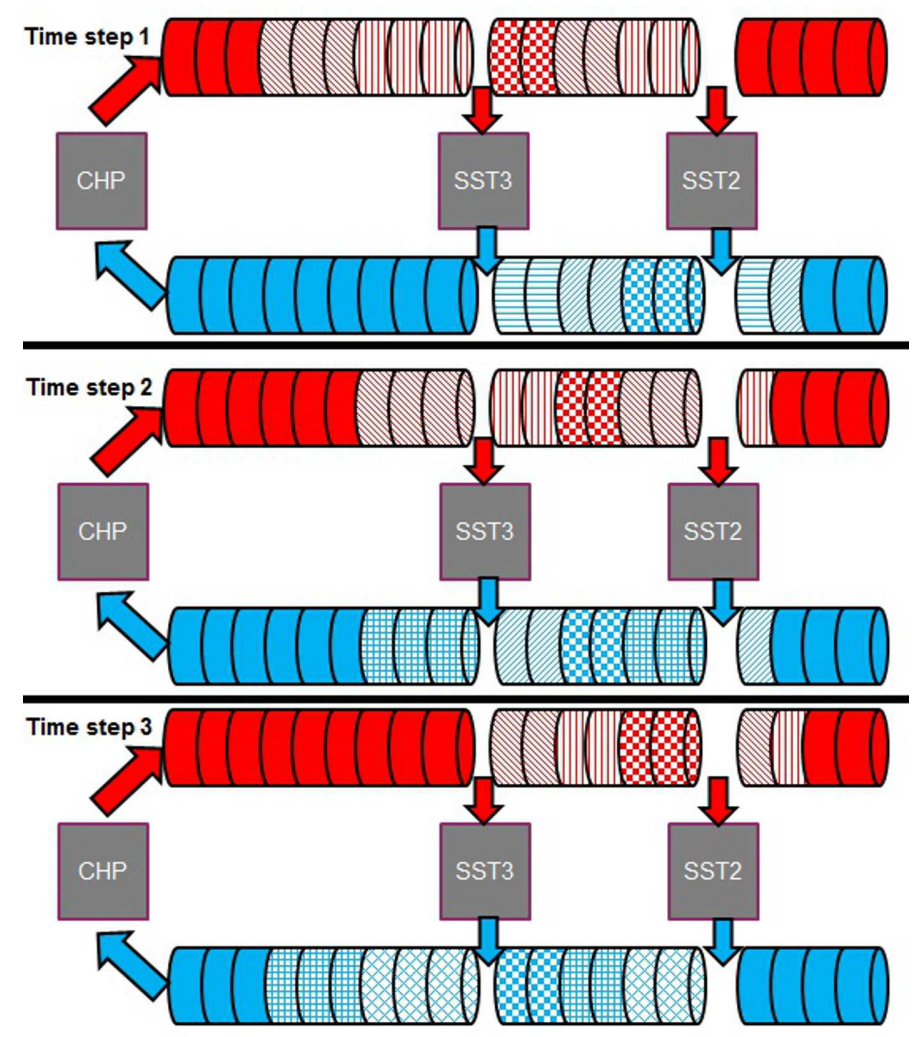

Figure 1: Principle of water wave travelling, separation and mixing.

to build an equivalent topology model which reflects the reality but omits or aggregates smaller pipelines.

\subsection{Modeling the network by delays, without topology}

Many DH networks are nowadays equipped with telemetry systems, providing measurements from substations and heat chambers in abundance. One of the possibilities of such systems is to trace the actual waves of supply water, by comparing the time at which a significant temperature change reaches particular points of the network. In this simple way, the time of water transportation between the heat source and each substation with measurement can be determined. The time of transportation is of course dependent on the total flow in the network, which in turn varies according 
mainly to the outdoor temperature. By calculating the time of transportation under different circumstances (i.e., at different total flows), for every significant point of the network (e.g., substation) a simple function defining the time of transport can be created.

Apart from comparing the time at which a significant change of supply temperature reaches particular points of the network, also the value of the temperature in the peak of change can be monitored. By looking at the difference between peak value at the heat source and peak value at a specific point of the network, temperature drop can be described for every point of the network by a function of outdoor temperature or total water flow, or other variables.

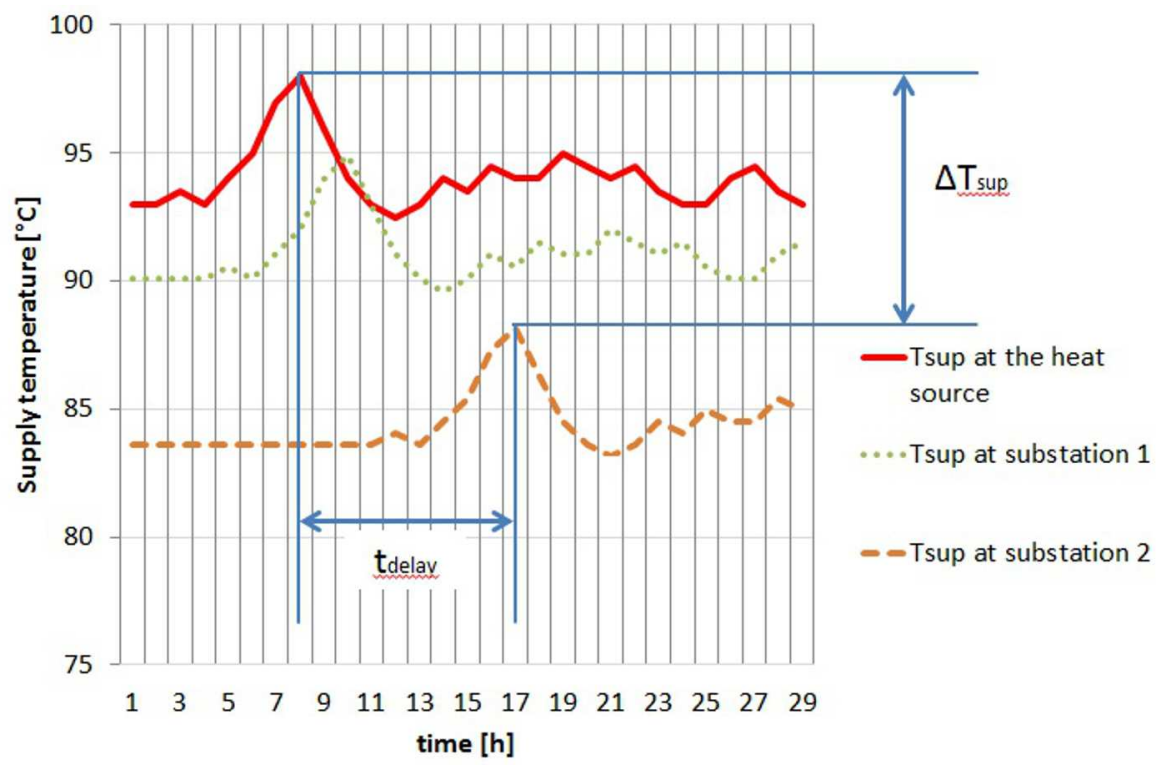

Figure 2: Illustration of finding the delay and temperature drop based on measurements of supply temperature at the heat source and at a substation.

Figure 2 illustrates the procedure of finding the delay and temperature drop for a substation. Applying this procedure many times, with measurements recorded under different circumstances, allows to build a set of equations describing transportation time and temperature drop for each significant point as a function of the circumstances which varied during tests. A set of such empirical functions can serve as a model of the network - without considering the topology of pipelines explicitly. 
The approach to creation of such functions has been described with the focus on supply lines. It can be hard to trace significant changes of return temperature, because of mixing of return water at every substation outlet. A simple solution is to assume that the transportation time is the same for return lines as for supply lines (which is in general true in most grids as they are typically symmetrical and the flows are almost equal in both directions), and that the temperature drop on return side is a constant fraction of the temperature drop on the supply side (for the same reasons). Proportions between the temperature drop of supply and return will be the same as the proportions of temperature difference between outdoor temperature and respective temperatures:

$$
\frac{\triangle T_{\text {sup }}}{\triangle T_{\text {ret }}}=\frac{T_{\text {sup }}-T_{\text {out }}}{T_{\text {ret }}-T_{\text {out }}}
$$

As soon as a set of formulas for all significant points of the network is ready, it can serve to model the thermal behavior of the grid without explicitly considering the topology of pipelines at all. For every substation, at every time step, the supply temperature can be calculated as

$T_{\text {sup }}^{S S T} i(t)=$

$$
T_{\text {sup }}^{\text {heat }} \text { source }\left(t-t_{\text {delay }}^{S S T} i\left[T_{\text {out }} \text {, day type, } \ldots\right]\right)-\triangle T_{\text {sup }}^{S S T} i\left[T_{\text {out }}, \text { day type, } \ldots\right] \text {, }
$$

where: $T_{\text {sup }}^{S S T} i$ - supply temperature reaching the substation $i$ in time $t$, $T_{\text {sup }}^{\text {heat }}$ source $[t]$ - temperature of supply at the heat source in time $t, t_{\text {delay }}^{S S T}{ }^{i}-$ time of water transport between the heat source and substation $i, \triangle T_{\text {sup }}^{S S T} i$ - temperature drop between the heat source and substation $i$ in supply pipelines (a function of outdoor temperature, type of the day, etc.).

Knowing the supply temperature at every important point of the network, it is possible to use the substation models and hydraulic models in order to calculate other parameters at those points. The supply temperature could be calculated more precisely with the use of total flow in the network rather than outdoor temperature; in such case the process of finding total flow and supply temperatures must be iterative. As soon as return temperatures at all substations are determined, the return temperature at the heat source can be calculated as an average of return temperatures at particular substations, weighted by their flows, and respecting the temper- 
ature drops and delays:

$$
\begin{aligned}
& T_{\text {ret }}^{\text {source }}(t)=\frac{\sum_{i=1}^{n} T_{\text {ret }}^{S S T i}\left(t-t_{\text {delay }}^{S S T}{ }^{i}\left[T_{\text {out }}, \text { day type, }, . .\right]\right) \dot{m}_{\text {flow }}^{S S T} i(t)}{\sum_{i=1}^{n} \dot{m}_{\text {flow }}^{S S T} i(t)} \\
& -\frac{\sum_{i=1}^{n} \triangle T_{\text {ret }}^{S S T} i\left[T_{\text {out }}, \text { day type }, \ldots\right] \dot{m}_{\text {flow }}^{S S T} i(t)}{\sum_{i=1}^{n} \dot{m}_{\text {flow }}^{S S T} i(t)},
\end{aligned}
$$

where: $T_{\text {ret }}^{\text {source }}(t)$ - temperature of return, reaching the heat source in time $t, T_{r e t}^{S S T} i(t)$ - temperature of return at the substation $i$ in time $t$, $\triangle T_{\text {ret }}^{S S T} i_{-}$- temperature drop between the substation $i$ and the heat source in return pipelines (a function of outdoor temperature, type of the day, etc.), $\dot{m}_{\text {flow }}^{S S T} i(t)$ - flowrate of $\mathrm{DH}$ water at substation $i$ in time $t$.

Return temperature at the heat source, together with the supply temperature and total flow, allows to determine the heating power of the heat source at every time step. Thus, it is possible to fully model the thermal behavior of the network including its inertia, without building any topology model - just based on the empirically measured delays and temperature drops.

As long as the total flow in the network is constant, the model based on delays gives exactly the same results as a 'wave' model (because both assume no mixing of the water and account for delays as well as temperature drops). However, if the total flow varies in time, the results given by the model based on delays reveal its main drawback. Unfortunately, unlike the other models presented so far, the model based on delays does not by definition respect the energy balance when applied in time steps. In reality, if the total water flow at an hour $X$ is higher than at preceding hours, then of course during the hour $X$ the heat source will be reached by the volume of water returning from substations, which has left these substations over a period of more than an hour. For example, if the flow at substation $i$ at hour $X$ is $1 \mathrm{t} / \mathrm{h}$, then on average $1 \mathrm{t}$ of water from substation $i$ will reach the heat source during that hour. Let us assume that the delay is $Y$, and the flow at substation $i$ at hour $X-Y$ was $0.9 \mathrm{t} / \mathrm{h}$. Only part of the water from substation $i$ reaching the heat source at hour $X$ will have the temperature determined by leaving the substation at hour $X-Y$. Other part will have a temperature resulting from leaving the substation at a later hour which may be different. The model does not consider that. As a result, an overheat of supply during one hour of low total flow can result in an overheat of return during an hour of high total flow. And that means artificial 
creation of energy in the model based on delays. This effect is significant if the flow changes are large, and may affect the results of optimizations in such cases.

\section{Case study - using the inertia of the network simulated with different thermal network models}

It is relatively simple to model the behavior of a DH network in steady state. All the models described above can give reasonable results for such situation. Most DH companies are used to relay on steady state calculations for the purposes of simulating the network development, designing the pipelines, sizing the heat sources, etc. However, operational optimization with load shifting is by definition connected to transient behavior of the network. In order to help in optimization of production planning utilizing the inertia of the network, a model must consider variations of supply temperature and their influence on power profile. As this is the key element, all the models described above have been compared in a case study, in order to analyze the way they handle the phenomenon of thermal energy storage in pipelines.

\subsection{Model of the network}

A simple model of a district heating network, comprising one heat source and three substations, has been created using different methods. The substations are connected in series. It is assumed that each of the substations consumes $10 \mathrm{MW}$ of heat all the time, and that the transportation delays are:

- 10 hours between the heat source and the substation 1 (furthest from the network),

- 6 hours between the heat source and the substation 2 (in the middle),

- 3 hours between the heat source and the substation 3 (closest to the heat source).

A network that corresponds to aforementioned assumptions is presented in Fig. 3. Four Microsoft Excel sheets have been created, each of them modeling that network with one of the approaches described before. Then the 
results of the same supply temperature variations, simulated with different models, have been compared.

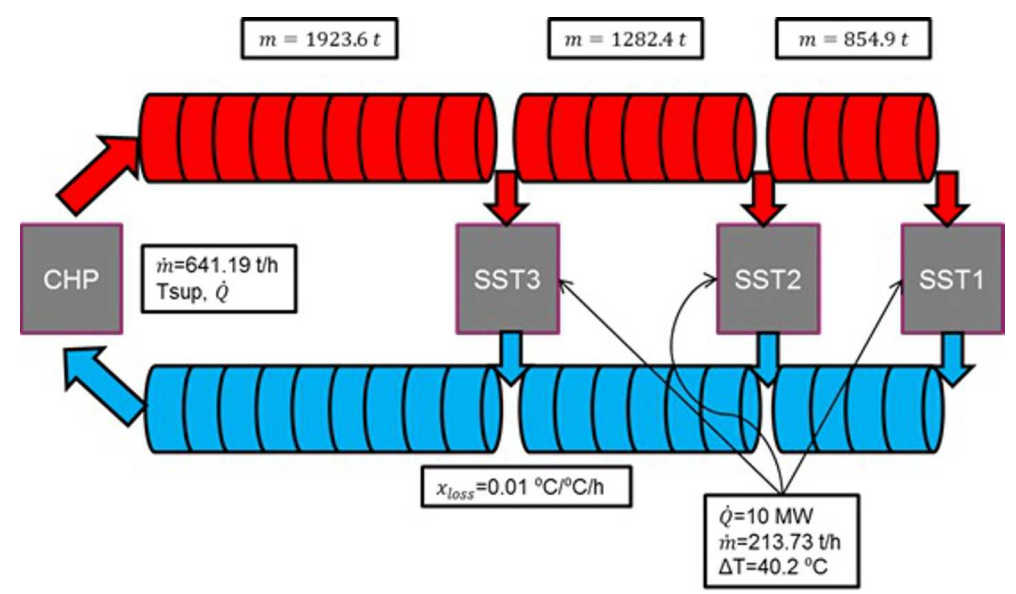

Figure 3: The simple network model built for case study.

\subsection{Single overheat}

A single overheat of the network, i.e., a change of supply temperature at the heat source from $93{ }^{\circ} \mathrm{C}$ to $97^{\circ} \mathrm{C}$ for 4 hours and then back to $93^{\circ} \mathrm{C}$, has been simulated with all of the models described before.

Water mixing model The results of simulation with water mixing model are shown in Fig. 4. Directly after the increase of supply temperature, total heating power at the CHP plant increases sharply and remains stable for 2 time steps. Then the return temperature starts to increase, causing the heating power to slightly decrease. At the moment when the supply temperature is decreased back to initial value (end of overheat), the heating power plummets below the initial value (because return temperature is high). For some short time the power keeps decreasing, as hotter water from substations reaches the plant. Then the heating power starts to slowly increase, as the return temperature decreases, at the end of the time horizon reaching almost the initial value. The changes of both supply and return temperatures at substations are moderate and time consuming, and the borders of the wave of hotter water are disappearing over time, especially on the return side. 


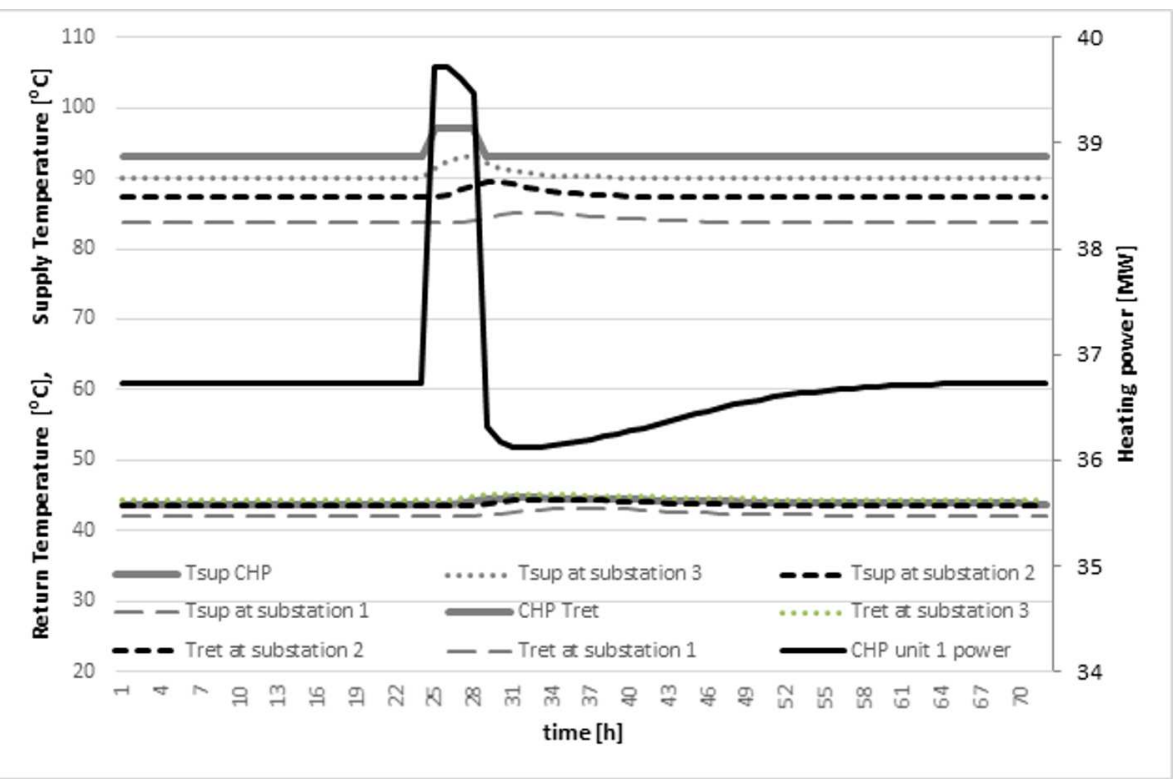

Figure 4: A single overheat of network, simulated with 'water mixing' model.

Whole network model The same case has been modelled with the approach treating whole network as one substation (with the heat demand of $30 \mathrm{MW}$, as the sum of all 3 substations) and one pipeline section (with the mass of $4060 \mathrm{t}$ of water, as the sum of all 3 sections). The results are shown in Fig. 5. The supply temperature at the 'substation' which represents all substations is about the average of 3 temperatures for particular substations simulated with 'water mixing' model. The return temperature is almost the same, and most importantly, the heating power resulting from this simulation is very close to the one resulting from simulation considering 3 pipeline sections and substations. All the changes take place in the same way, and in fact the values of heating power at particular time steps differ only by up to $0.27 \%$. It shows that as long as the purpose of modeling is to simulate the reaction of the network in order to know how much energy can be stored by a given overheat, in the 'water mixing' approach, aggregation of substations does not significantly affect the accuracy. Only if knowing the temperatures or flows at particular substations is necessary (e.g., because of constraints applied there), it is necessary to model them separately, because the supply temperatures depend to a large extent on the distance from heat source. 


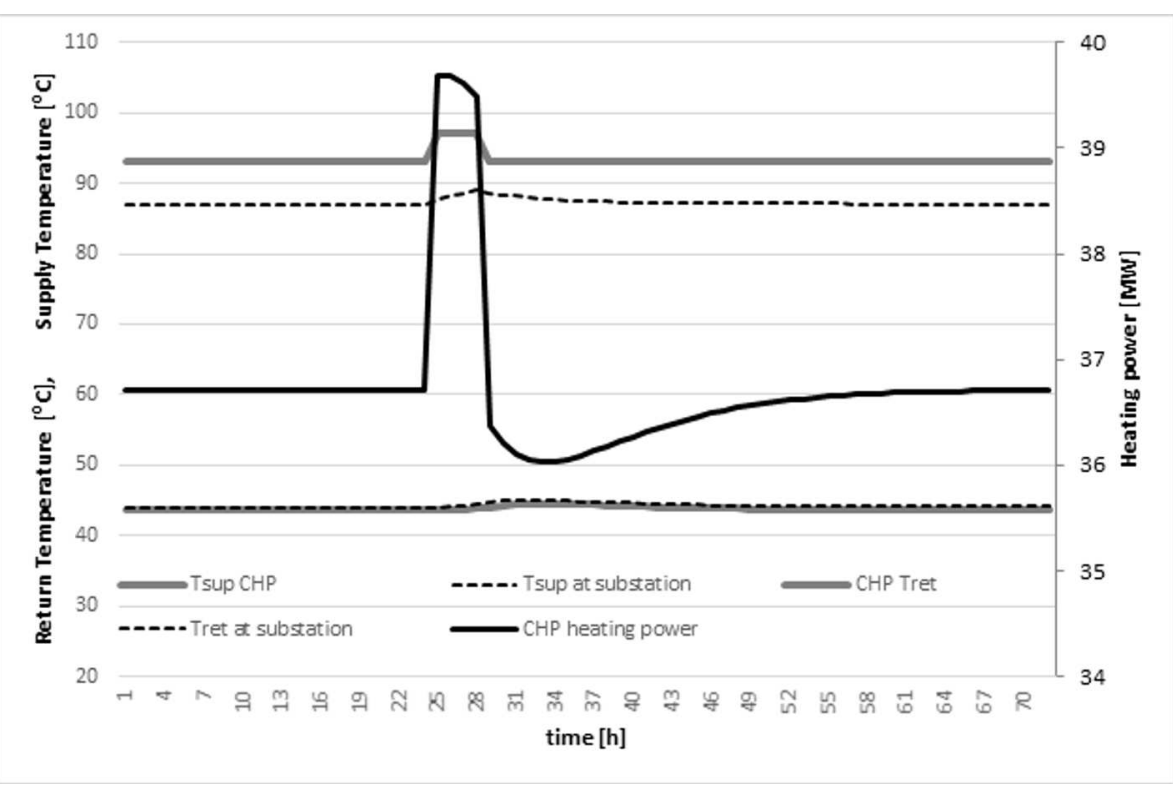

Figure 5: A single overheat of network, simulated with 'whole network' model.

Wave model Figure 6 presents the results of simulation with the 'wave' model. These results are significantly different from the results of 'water mixing' model. The hotter water propagates in the supply lines as a 'wave' with clear borders, so that the shape of supply temperature profile at every substation is the same (only the delay and the temperature drop differ). Similarly, the hotter water in the return lines propagates back to the source in the form of 'waves'. At some points of the network, multiple 'waves' can be observed - first resulting from increased temperature at the closest substation, then from increased temperature of water that left some further substation and passes by. As long as the delays between substations are longer than the duration of overheat, the waves on return side do not overlap and the first wave on the return side does not come to the heat source before the end of overheat. As a result, during the overheat the heating power of CHP plant is stable, higher than the initial heating power. After the overheat it drops back to the initial value, and then decreases further as soon as first wave of hotter water reaches the return side of CHP plant. Later the heating power goes back to the initial value, and drops again when next waves of hotter return water reach the plant. The first decrease of heating power below initial value is the biggest, because it results from 
the first wave. The first wave represents the water volume from the closest substation, which spent the smallest amount of time in the network, and for this reason its additional temperature drop is smallest. So it contains hotter return water than the waves that follow, in turn resulting in smaller temperature difference at the heat source which leads to smaller heating power.

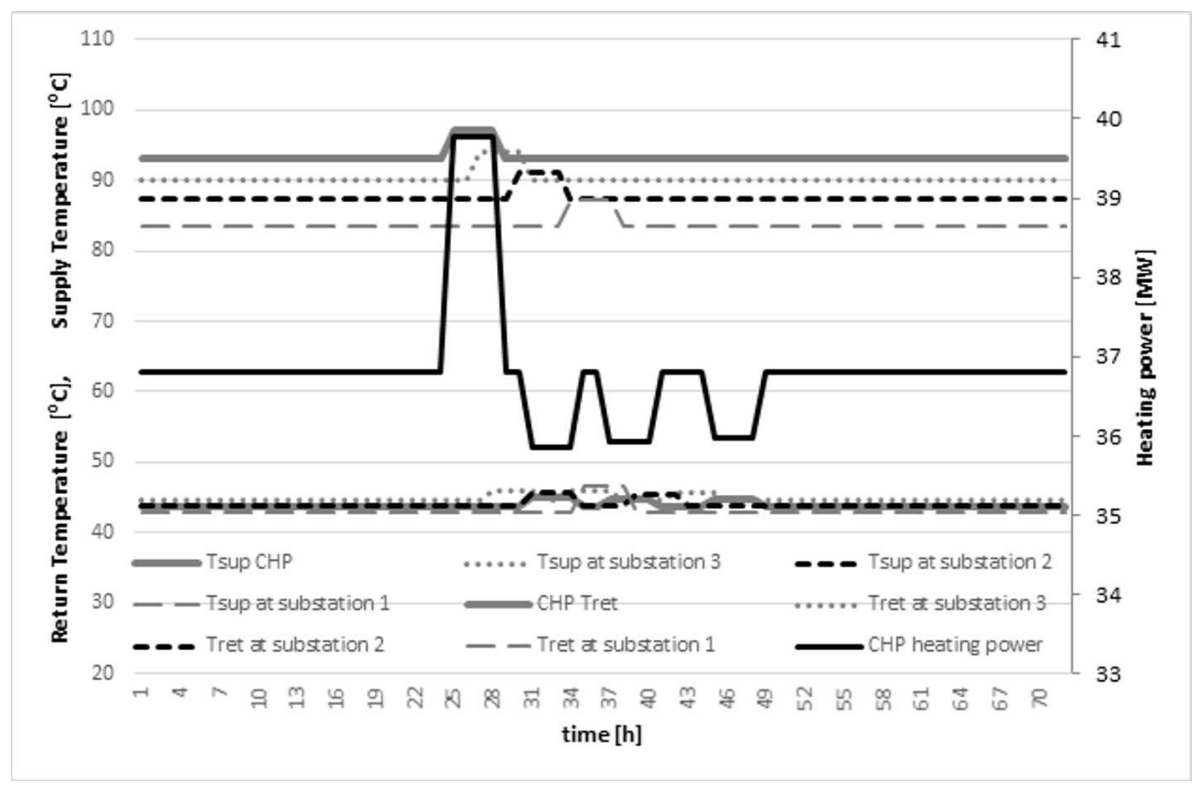

Figure 6: A single overheat of network, simulated with 'wave' model.

Delays model As it has been predicted in the theoretical part, the model based on delays has given exactly the same results as the 'wave model'. It proves that these two models are identical, as long as total flow is constant. And when it is not, the 'delays' model does not respect the energy balance. For these reasons the 'delays' model was not investigated further. It remains in fact a simpler way of defining the wave model for constant flows, and a solution which should not be used for cases where flows are significantly variable. 


\subsection{Long overheat}

A long overheat of the network, i.e., a change of supply temperature at the heat source from $93^{\circ} \mathrm{C}$ to $97^{\circ} \mathrm{C}$ for 16 hours and then back to $93^{\circ} \mathrm{C}$, has been simulated with the same models as in case of the single overheat. The results given by 'water mixing' and 'whole network' model are shown in Figs. 7 and 8, respectively. The profiles of heating power and DH water temperatures are analogical to the ones simulated for a short overheat. They are also very similar to each other, with the difference between heating power in both models limited to $0.25 \%$.

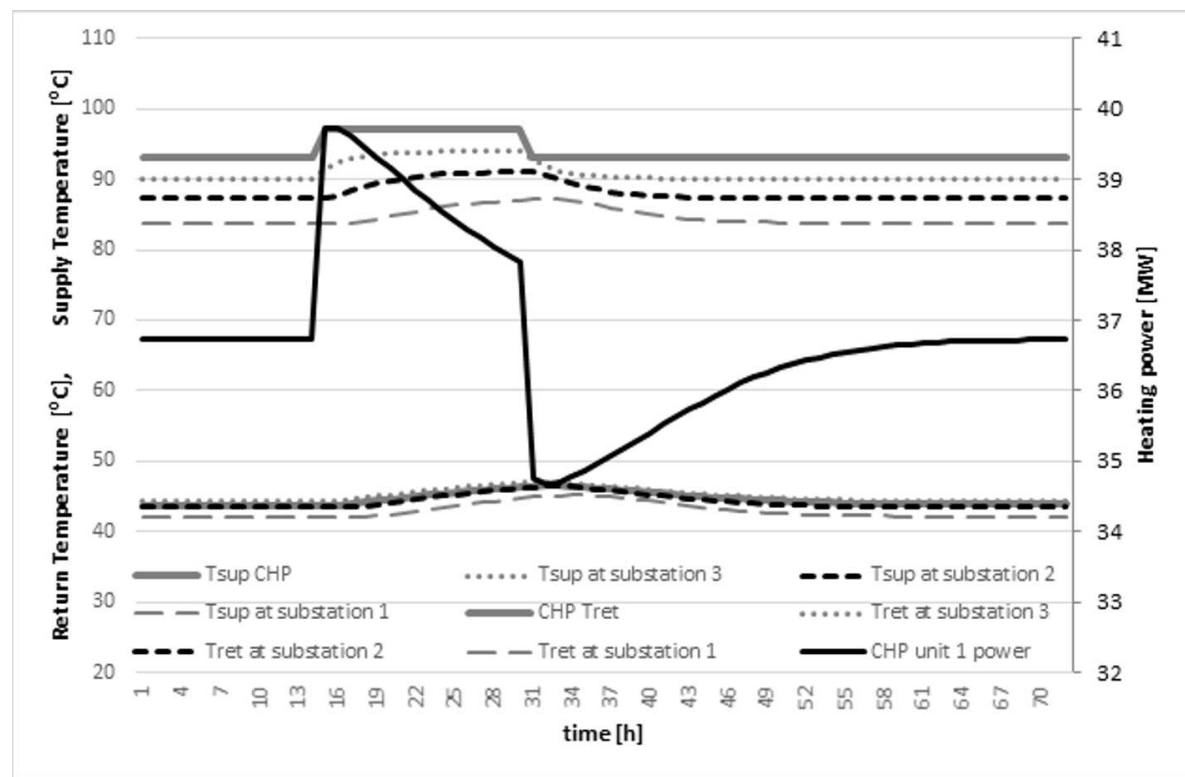

Figure 7: A long overheat of network, simulated with 'water mixing' model.

Figure 9 illustrates the results given by the 'wave' model (identical with the results from a 'delays' model). In this case, the 'waves' overlap, so the heating power during the overheat is not stable. It drops when hotter return water from the closest substation reaches the plant, and then it drops again when hotter return water from second substation reaches the plant. Then after the very large decrease due to end of overheat, the power drops for the last time when hotter return water from the third substation reaches the plant. Soon after that, the heating power increases in three steps, as three waves of hotter return water from particular substations end. 


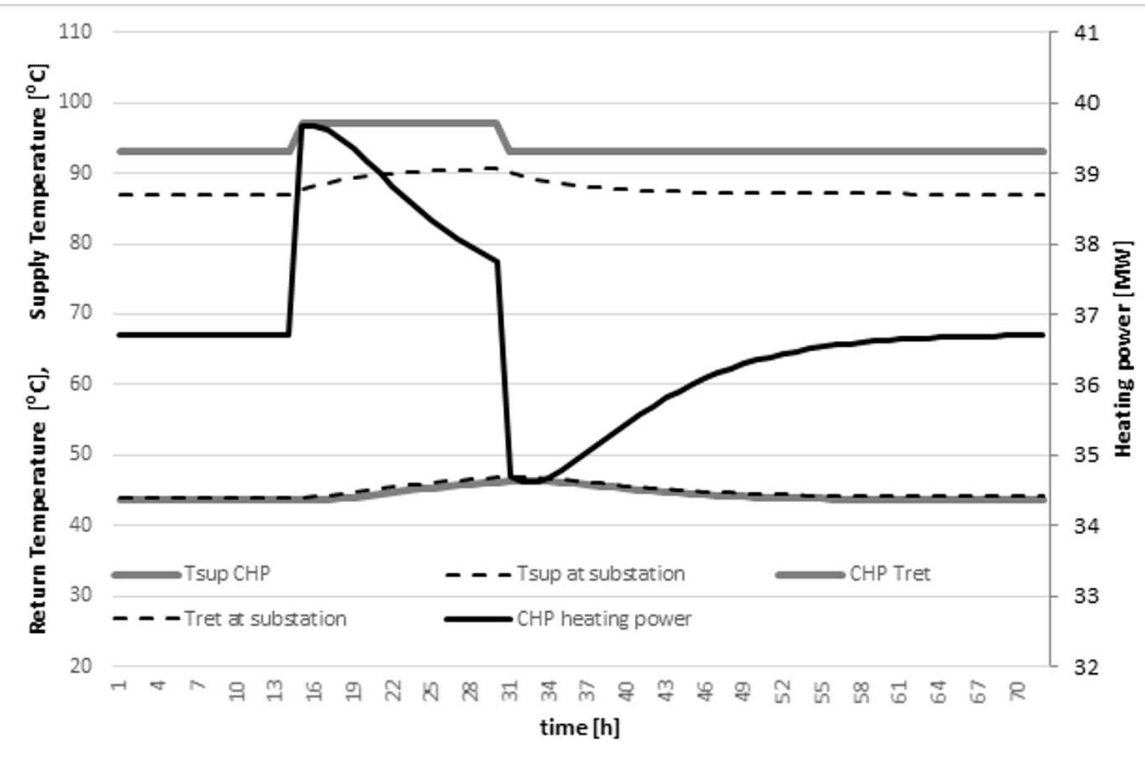

Figure 8: A long overheat of network, simulated with 'whole network' model.

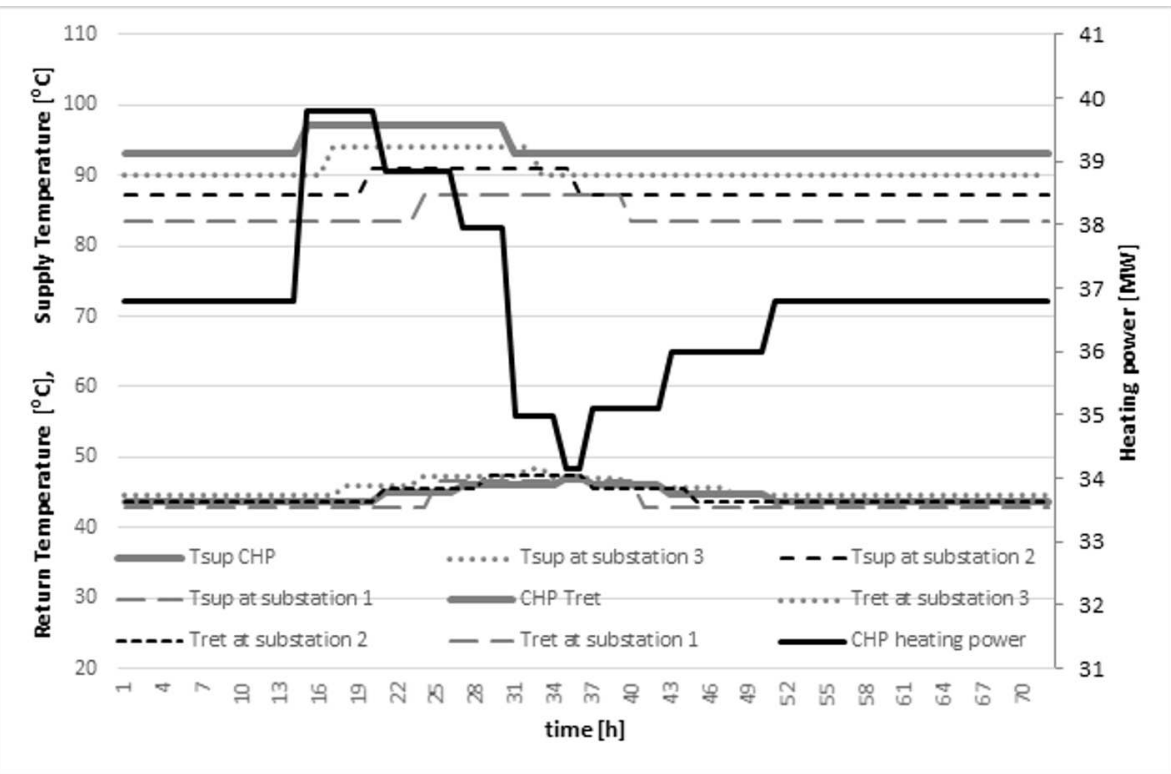

Figure 9: A long overheat of network, simulated with 'wave' model. 
It is interesting, that for longer overheats, the results of 'water mixing' model and 'wave' model are less different. The steps in which heating power increases and decreases, when they are not separated by periods of heating power reaching the initial value, resemble the continuous way in which heating power changes according to the 'water mixing' model. This effect of similarity will obviously grow as the number of substations (and pipeline sections) in both models grows.

\subsection{Two overheats}

Two overheats of the network, i.e., a change of supply temperature at the heat source from $93^{\circ} \mathrm{C}$ to $97^{\circ} \mathrm{C}$ for 8 hours, then back to $93^{\circ} \mathrm{C}$ for 6 hours, again to $97^{\circ} \mathrm{C}$ for 8 hours and then back, have been simulated with the same models. The results given by 'water mixing' and 'whole network' model are shown in Figs. 10 and 11, respectively. Again, they are very similar to each other, with the difference between heating power in both models limited to $0.39 \%$. Each of the overheats resembles a single overheat, and their effects add without any unexpected interaction.

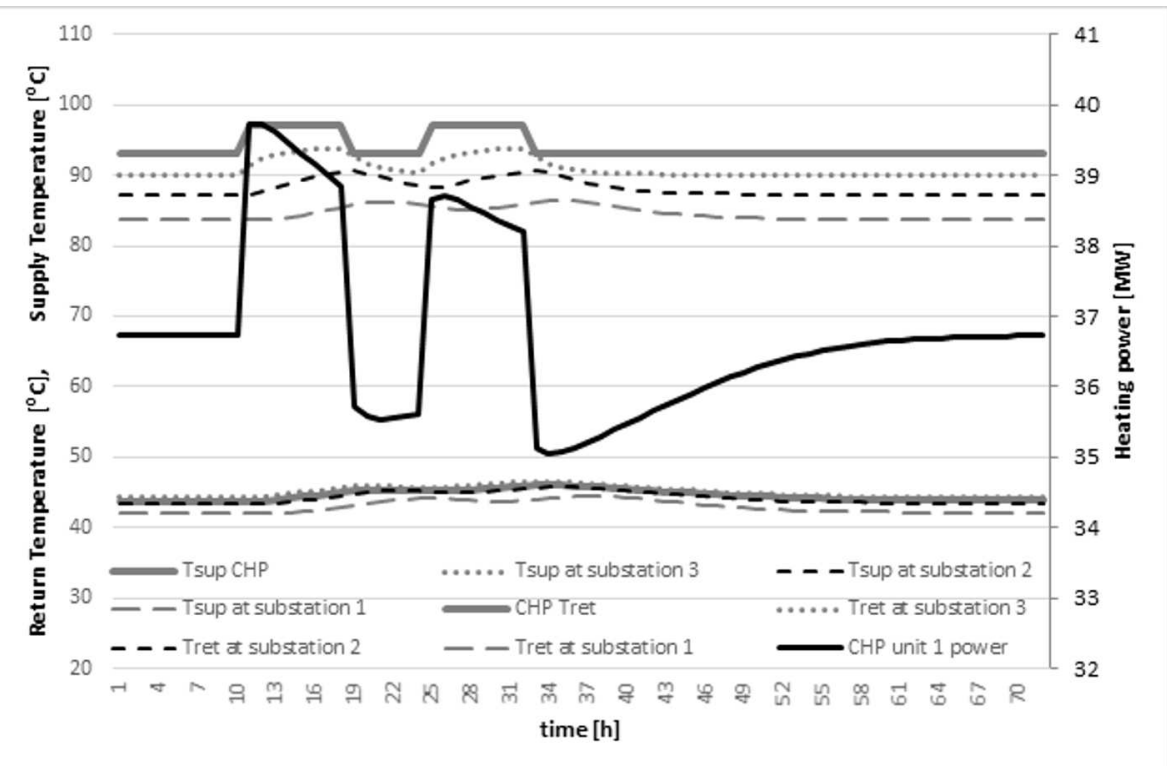

Figure 10: Two overheats of network, simulated with 'water mixing' model. 


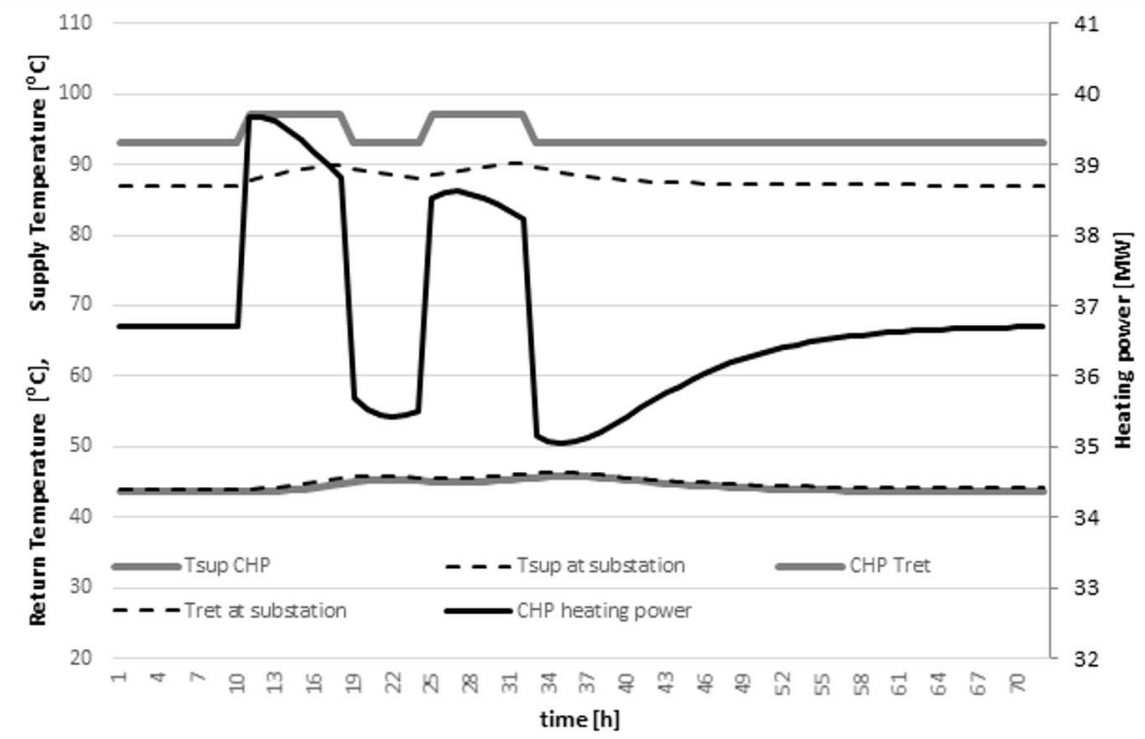

Figure 11: Two overheats of network, simulated with 'whole network' model.

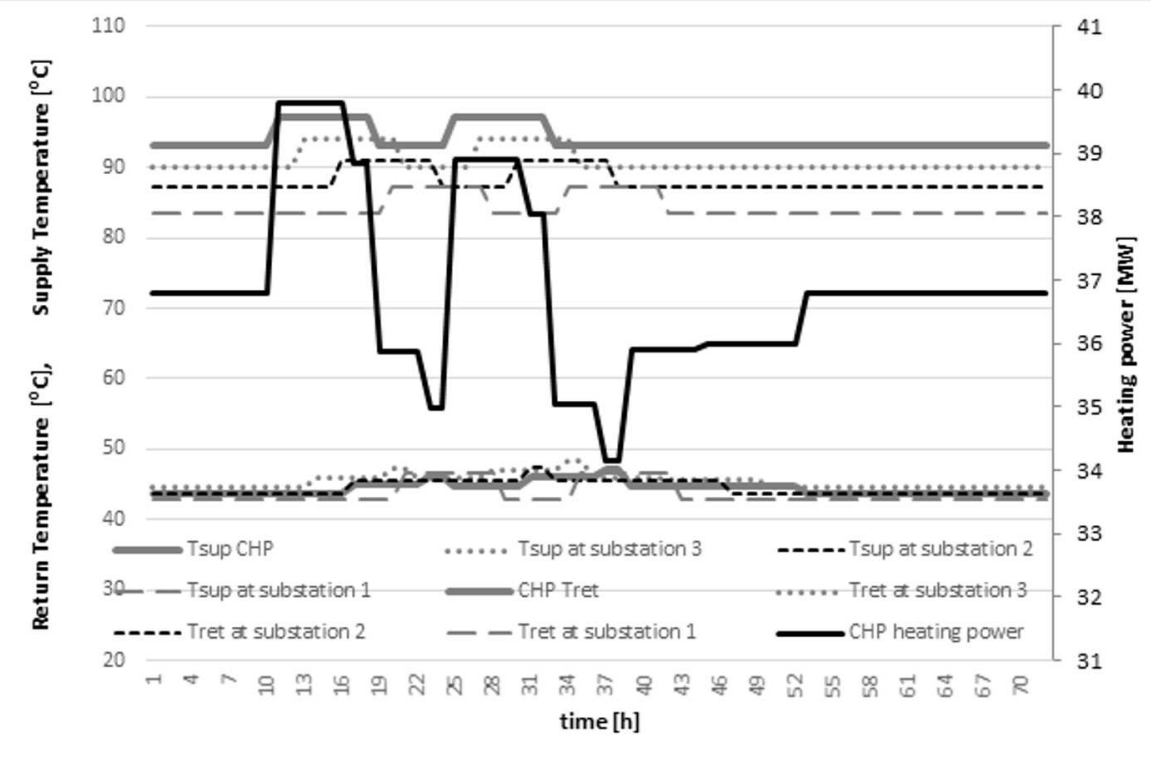

Figure 12: Two overheats of network, simulated with 'wave' model. 
Figure 12 presents two overheats simulated with the 'wave' model (identical with the results from a 'delays' model). It can be seen, that in this case particular waves overlap, resulting in a complicated shape of heating power profile. However, in general this profile resembles the profiles simulated with 'water mixing' models. Both overheats have direct, significant effect on the heating power, which then weakens as the responses from substations reach the plant. In the end, the temperature of water in all network sections comes back to initial value, which of course leads to the heating power also coming back to initial value.

\subsection{Conclusions from the case study}

The case study has confirmed, that for constant total flow, the 'delays' model is identical with the 'wave' model, and the 'whole network' model gives almost the same results as the 'water mixing' model (from the point of view of the heat source).

The 'wave' model correctly simulates the propagation of hot water waves in the supply lines and its assumptions seem to be closer to the reality. However, its results seem to be artificial when only a few substations are modelled. The number of substations is reflected by the number of heating power drops after the overheat, which are in case of a short overheat separated by periods of heating power equal to initial value, which seems artificial. The 'wave' model is also the most complicated, difficult to create and time consuming to calculate.

The 'water mixing' model is simpler and faster than the 'wave' model. The changes simulated with it are more moderate and continuous, which in general reflects the reality well. However, it fails to simulate the way in which hot water waves propagate in the supply lines.

Both 'water mixing' and 'wave' model have a potential to be calibrated in a way which will make them reflect the reality well. What is more important, it seems that the differences between results of particular models tend to decrease as the number of substations grows and as the duration of overheat increases. For this reason, it is possible to perform optimization using any of these models, or a mixture of them. The decision, which one to use, should be taken for particular cases, based on the characteristic of the $\mathrm{DH}$ network, the possibilities and requirements regarding programming and time of calculations. For testing purposes, simpler and faster 'water mixing' model can be enough to perform calculations and draw conclusions. Continuity of its results also allows to make simulations with few substa- 
tions in the model, without risking that some of the effects are artificial and unrealistic (like in the case of short overheat).

\section{Summary}

The problem of operational optimization in modern district heating networks has been outlined. Among new solutions, thermal load shifting with the use of inertia of the networks has been chosen for analysis. Two approaches to modeling of transient behavior of a network have been presented. The results of their application have been compared, advantages and disadvantages have been identified. Presented models can be used as a part of future solutions for optimization of district heating systems' operation, sometimes referred to as smart district heating.

Acknowledgement The research described in current paper has been conducted in cooperation with Veolia Energia Polska, as part of PhD program.

Received 18 October 2017

\section{References}

[1] Viana A., Pedroso J.P.: A new MILP-based approach for Unit Commitment in power production planning. IEEE Trans in Power Systems, 2012.

[2] BADYDA K: Mathematical model for digital simulation of steam turbine set dynamics and on-line turbine load distribution. Transactions Inst. Fluid-Flow Mach. 126(2014), 65-82.

[3] Szapajko G., Rusinowski H.: Mathematicalmodeling of steam-water cycle with auxiliary empirical functions application. Arch. Thermodyn. 31(2010), 3, 165-183.

[4] Rusinowski H., Szapajko G.: Energy evaluation of steam-water cycle operation with mathematicalmodeling application. Arch.Thermodyn. 32(2011), 4, 101-117.

[5] Fonseca J.G.S. JR, Schneider P.S.: Simulation of a thermal power plant with district heating: Comparative results of 5 different codes. Energy 31(2006), 19551968.

[6] Ziębik A., Szegda D., Qvale B., ElmegaARd B.: Thermodynamic simulation analysis of a multifuel CHP plant basing on the technological diagram of Avedore unit 2. Arch. Thermodyn. 31(2010), 1, 79-93.

[7] Bujalski W.: Optimization of the operation of a CHP plant equipped with a heat accumulator. Oficyna Wydawnicza Politechniki Warszawskiej, Warszawa 2013 (in Polish). 
[8] Fazlollahi S., Beckera G., Maréchal F.: Multi-objectives, multi-period optimization of district energy systems: III. Distribution networks. Comput. Chem. Eng. 66(2014), 82-97.

[9] ZięBik A., GŁadysz P.: Optimal coefficient of the share of cogeneration in the district heating system cooperating with thermal storage. Arch. Thermodyn. 32(2011), $3,71-87$.

[10] Milewski J., Wolowicz M., Bujalski W.: Seasonal thermal energy storage a size selection. Appl. Mech. Mater. 467(2014), 270-276.

[11] Converse A.O.: Seasonal Energy Storage in a Renewable Energy System. In: Proc. of the IEEE 100(2012), 2, 401-409.

[12] Zucker G., Palensky P., Judex F., Hettfleisch C., Schmidt R., Basciotti D.: Energy aware building automation enables Smart Grid-friendly buildings. Elektrotechnik \& Informationstechnik 129(2012), 4.

[13] LeŚKO M. BujALSKI W.: Operational optimization in district heating systems using thermal inertia of buildings. Rynek Energii (125( 2016), 4.

[14] Stevanovic V.D., Prica S., Maslovaric B., Zivkovic B., Nikodijevic S.: Efficient numerical method for district heating system hydraulics. Energ. Convers. Manage. 48(2007), 5, 1536-1543.

[15] Oppelt T., Urbaneck T., Gross U., Platzer B.: Dynamic thermo-hydraulic model of district cooling networks. Appl. Therm. Eng. 102(2016), 336-345.

[16] Basciotti D., Judex F., Pol O., Schmidt R.: Sensible heat storage in district heating networks: a novel control strategy using the network as storage. Austrian Institute of Technology, Energy Department, Sustainable Building Technology. https://www.researchgate.net/publication/260384884_Sensible_heat_storage_in _district_heating_networks_a_novel_control_strategy_using_the_network _as_storage, (accessed 21.11.2017).

[17] Benonysson A. et al:: Operational optimization in a district heating system. Energy Convers. Mgmt 36(1995), 5, 297-314.

[18] Kamler W.: Heating Technology. PWN, Warszawa 1979 (in Polish). 